\title{
NutOrMed-optimising nutrition, oral health and medication for older home care clients-study protocol
}

\author{
Miia Tiihonen ${ }^{1,2^{*}}$, Kirsi Autonen-Honkonen ${ }^{3}$, Riitta Ahonen ${ }^{2}$, Kaija Komulainen ${ }^{3}$, Liisa Suominen ${ }^{3,5}$,
} Sirpa Hartikainen ${ }^{1,2}$ and Irma Nykänen ${ }^{1,2,4}$

\begin{abstract}
Background: Malnutrition, polypharmacy and poor oral health are major concerns among older people living at home, and they contribute to disability and care dependency. This article describes the study protocol of the Nutrition, Oral Health and Medication (NutOrMed) intervention study.

Methods/design: This study was a population-based multidisciplinary intervention study. The NutOrMed study sample included home care clients aged 75 years or over living in Eastern and Central Finland. All the participants $(n=275)$ were interviewed at home at the baseline and after a 6-month intervention. The primary outcomes are nutritional status, oral health status and factors associated with them. These measures were administered at the baseline and after a 6-month follow-up. Agreement between in-home interviews and electronic medical records regarding drug use was checked at the baseline.

Discussion: Our study provides evidence about factors associated with nutrition, oral health and medication that may compromise the ability to stay at home. The strengths of this study are its multidisciplinary approach, the diversity of the measures, including interviews and clinical examinations, and the multi-interventions targeting those in need.

Trial registration: ClinicalTrials.gov: NCT02214758

Keywords: Home care, Nutrition, Oral health, Medication, Study protocol
\end{abstract}

\section{Background}

The European population is ageing due to the increased number of older people aged 75 years or over [1]. Nine out of ten people aged 75 years or older would like to live in their own home instead of living in residential care [2]. This presents a growing need for homedelivered services. Nowadays in Finland, $11.9 \%$ of people aged 75 years or older are municipal home care clients [3]. The purpose of home-delivered services is to support maintaining of health, functional abilities and quality of life [4]. These services and assistance at home should support older patients' needs [2].

\footnotetext{
* Correspondence: miia.tiihonen@uef.fi

'Kuopio Research Centre of Geriatric Care, University of Eastern Finland, P.O.B 1627, FI-70211 Kuopio, Finland

${ }^{2}$ School of Pharmacy, Faculty of Health Sciences, University of Eastern Finland, P.O.B 1627, FI-70211 Kuopio, Finland

Full list of author information is available at the end of the article
}

Malnutrition, excessive polypharmacy and poor oral health are major risks to the health and functional abilities of older people living at home $[5,6]$. The prevalence of possible malnutrition-measured by the Mini Nutritional Assessment SF (MNA-SF) test-is $15 \%$ among older community-dwelling persons [7] and about $50 \%$ among older home care clients in Finland [8]. Poor oral health is common among older people receiving home care services in Japan [8] and Sweden [9]. Malnutrition affects oral health, and poor oral health, in turn, may lead to malnutrition $[7,10]$. In addition, home care clients have several diseases, and the prevalence of polypharmacy (defined as use of six or more medications) is $51 \%$ [11]. Polypharmacy and malnutrition may influence each other, and the interaction between a larger number of drugs and nutritional status is currently not fully appreciated. 
Previous studies have mainly focused on the general community-dwelling [7, 12, 13] older population or persons living in residential care [8, 14]. As far as we know, no studies of the vulnerable home care population have examined the relationship between nutrition, oral health and medication use. A comprehensive perspective is important because home care clients are more vulnerable and at a higher risk of having adverse health outcomes. Here, we describe the study protocol of the Nutrition, Oral Health and Medication (NutOrMed) intervention study.

\section{Methods/design Study design}

This study was a prospective non-randomised populationbased multidisciplinary intervention study.

\section{Participants and setting}

The NutOrMed study sample included home care clients aged 75 years or over living in Eastern and Central Finland (Fig. 1). The intervention group consisted of a random sample of 250 home care clients from community I with
105,141 inhabitants. The control group was comprised of a random sample of 75 people from community II with 20,224 inhabitants and a total sample of 115 people from community III with 7524 inhabitants. The intervention city was big enough to allow us to get a random sample, but the other two towns were smaller, and therefore, both towns were needed as control groups to maximise the number of controls. For the same reason, all the home care clients in community III had the possibility to participate (total sample). To avoid contamination, the intervention group was situated approximately $100 \mathrm{~km}$ away from the towns of the control groups. Randomisation inside communities I and II was done with a coded list of home care clients and an SPSS random sample tool. The study was introduced to the persons included in the sample by home care nurses both verbally and with a written bulletin. After that, those willing to participate gave their written consent. In the case of cognitive impairment assessed by a geriatrician, consent was given by a family member or trustee.

The sample size calculation was estimated based on Mini Nutritional Assessment (MNA) with the objective

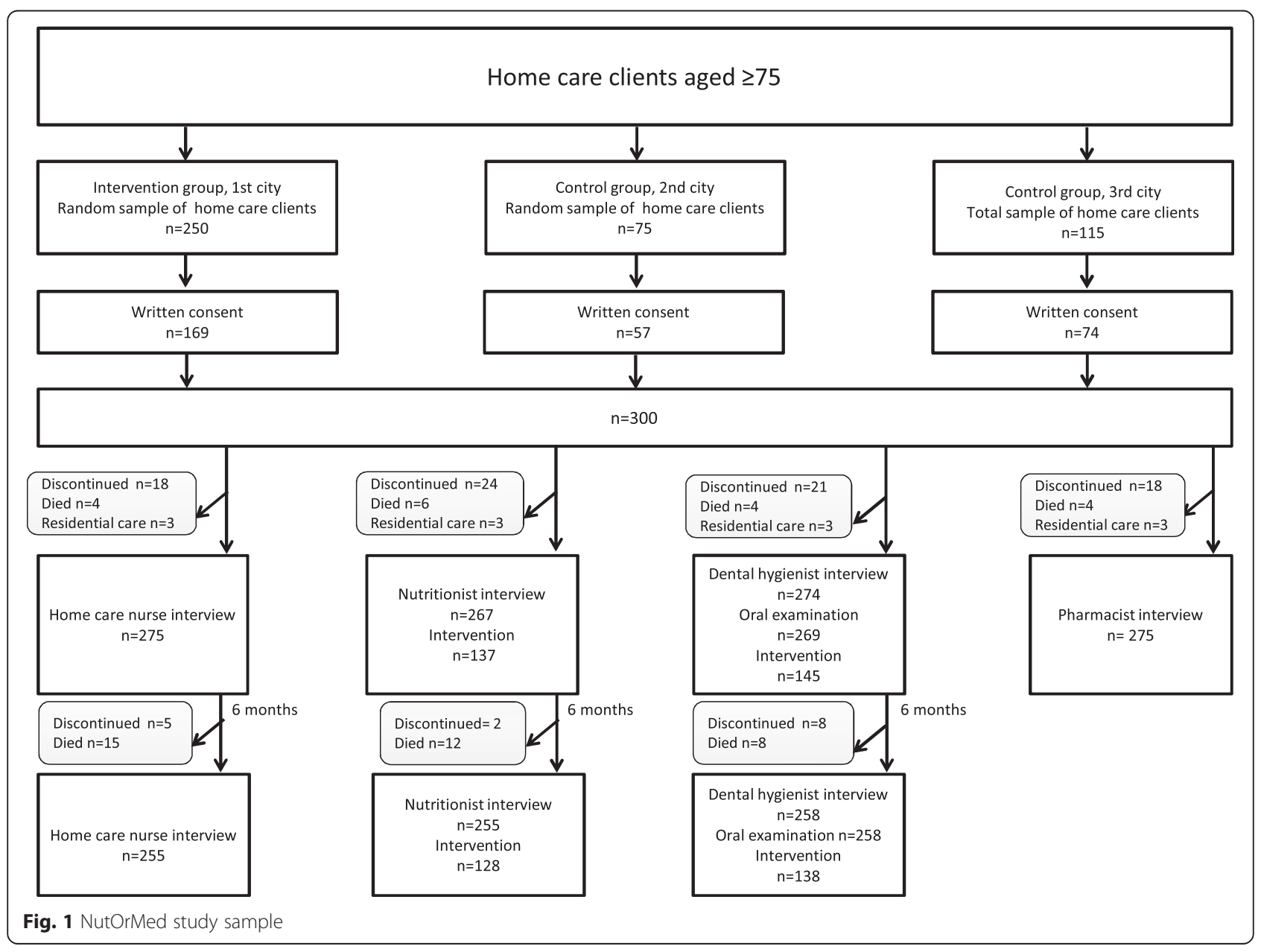


to detect an increase in MNA of 0.9 in the intervention group. In our previous study, the standard error of the mean change in MNA was 0.36 and the standard deviation, 3.0 [15]. The former study had a population (risk of malnutrition) comparable to the one in this study, so it was assumed that the standard error and standard deviations are also comparable. Furthermore, a power of $80 \%$ and a two-sided alpha of 0.05 were used. Based on these assumptions, 80 people are needed in each group. At the baseline, all the participants were interviewed at home by trained nurses, nutritionists, dental hygienists and pharmacists. We had no exclusion criteria regarding maximum age, morbidity or cognition. If a home care client was unable to reply correctly, mostly because of cognitive impairment, data collection was supplemented by an interview of a caregiver or a nurse.

\section{Baseline}

\section{Home care nurse interview and clinical examination}

Sociodemographic factors, health status, depression, cognitive functioning and functioning in the activities of daily living and instrumental activities of daily living were determined through interviews by home care nurses. The home care nurses were those who took care of each home care client on a daily basis. The nurses were trained by one person (IN) from the University of Eastern Finland. Cognition was assessed by the MiniMental State Examination (MMSE) on a scale of 0-30 with higher scores indicating better function [16], and mood was measured by the 15 -item Geriatric Depression Scale (GDS-15) [17]. Activities of daily living (ADL) were assessed using the 10-item Barthel Index [6], and instrumental activities (IADL), by the 8-item Lawton and Brody scale [18]. Scoring for the ADL index is from $0-100$ and for the IADL scale, from $0-8$, with higher scores indicating better functioning. Comorbidity was computed using a modified version of the Functional Comorbidity Index (FCI) [19]. Data on the following 13 medical conditions were available [20]: (1) rheumatoid arthritis and other inflammatory connective tissue diseases, (2) osteoporosis, (3) diabetes, (4) chronic asthma or chronic obstructive pulmonary disease (COPD), (5) coronary artery disease, (6) heart failure, (7) myocardial infarction, (8) stroke, (9) depressive disorder, (10) visual impairment, (11) hearing impairment, (12) Parkinson's disease or multiple sclerosis and (13) obesity (body mass index $(\mathrm{BMI})>30)$. The presence of each of the 13 conditions gave one point, and a higher FCI sum score represented greater comorbidity.

Biochemical measurements relevant to nutritional status, e.g. serum albumin and prealbumin, were performed. Serum albumin is considered the most accurate indicator of a patient's protein store and is therefore the main indicator of depletion of body protein stores, indicating protein malnutrition [21, 22]. The half-life of albumin is approximately 20 days, so it represents protein nutritional status from the previous weeks or month. Although there is no national standard or consensus, a serum albumin level of $3.5 \mathrm{~g} / \mathrm{dl}$ is usually considered low. There are also other reasons for low serum albumin levels. These are acute health disasters like septic infection and nephrotic syndrome, which can lower serum protein levels. Prealbumin has a half-life of 1 to 3 days; therefore, levels respond quickly to protein deprivation and nutritional intervention [23]. It has become a vital objective tool in evaluating acute nutritional changes. Prealbumin is considered a more reliable indicator of the patient's nutritional status. Prealbumin levels respond rapidly to nutritional support and are often used as an indicator of the effectiveness of a feeding intervention.

Orthostatic blood pressure (BP) was measured after $10 \mathrm{~min}$ of rest lying in bed. BP was measured in lying, sitting and standing positions (at 1 and $3 \mathrm{~min}$ ) by a trained nurse using an automated blood pressure monitor. A modified chair stand test [24] was used to assess the ability of the participants to perform sit-to-stand and stand-to-sit tasks five times as fast as possible. As a modification of the original test, the hands were held to each side and the participants were allowed to help with their hands, if needed. Time was measured with a stopwatch.

\section{Nutritionist interview and clinical examination}

Nutritional status and eating habits were evaluated by a nutritionist. She examined weight, height and daily eating routines with a 24 -h diary. Body weight was measured using a balance scale and BMI was computed as the ratio of weight to the square of height $\left(\mathrm{kg} / \mathrm{m}^{2}\right)$. Nutritional status was evaluated with an MNA test [25] and nutrient intake by using $24-\mathrm{h}$ dietary recalls. The MNA test is a validated and standardised screening tool developed to detect nutritional problems in older people. The MNA test fulfils many criteria for screenings instruments and definitive assessment tools. The MNA test was developed and validated on large representative samples of older persons, and it has been shown to be clinically useful [26]. The MNA test is the reference instrument recommended by The European Society for Clinical Nutrition and Metabolism (ESPEN) [27]. With a clinical examination as the gold standard, the sensitivity and specificity of the MNA test are 96 and $98 \%$, respectively. The maximum sum score of the MNA test is 30 ; scores of 24.0-30.0 indicate normal nutritional status, scores of 17.0-23.5, a risk of malnutrition and $0-16.5$, malnutrition. We used a $24-\mathrm{h}$ dietary recall. The nutritionist asked the participant to recall all food and drink during the previous $24 \mathrm{~h}$. She administered a 24-h 
recall via paper records to estimate energy and macronutrient (protein, carbohydrates and fat) intake.

\section{Dental hygienist interview and clinical examination}

Trained dental hygienists interviewed the participants prior to a clinical oral health examination based on written, patterned instructions. The interview first included questions about use of services (treatment place during the last visit to dental care, time since the last visit to dental care, reason for not having visited dental care) and self-perceived need for care. Next, the participants were asked about self-reported oral health (perceived oral health, pain and discomfort related to teeth or dental prosthesis, oral health-related quality of life, eating problems caused by teeth, sense of dry mouth), presence of a removable dental prosthesis, oral health-related behaviour (tooth brushing frequency, use of tooth cleaning devices, cleaning and overnight storage of the removable dental prosthesis, cleaning of oral mucosa) and problems related to cleaning of teeth and the mouth.

The clinical examinations were also conducted at the participant's home according to WHO instructions [28] and with help of a mouth mirror, a WHO periodontal probe (natural teeth) and a headlamp. The participants were either sitting or lying down. The subjects were first asked about the need for antibiotic prophylaxis in dental care, and if they answered yes, periodontal measurements were excluded. First the type and location (full or partial denture separately in the upper and lower jaws), need for repair (yes/no) and hygiene (good if plaque or tartar not shown) of the removable dental prosthesis were examined outside the mouth. Possible pain related to use of a prosthesis was also asked. The intraoral examination began with an examination of the oral mucosa using a dental mirror and gauze. After this, the number, location and condition of the teeth were recorded as follows: sound, filled with no need for repair, fractured with no caries, carious, root caries, radix without caries, missing or not able to examine. Tooth mobility was defined with one finger and an instrument and registered as none, 1-2 teeth with mobility or 3 or more teeth with mobility. Spaces in the dental arch were recorded separately in the upper and lower jaw by sextants as follows: none, space present but filled by a removable prosthesis, fixed prosthesis or implant and space present but not filled. Presence of plaque on each tooth was registered according to the modified Silness \& Löe [29] index and recorded as none, found at the gingival margin only, found also elsewhere or not able to examine/ tooth missing. Presence of gingival bleeding on probing was measured for each teeth except for wisdom teeth and recorded as yes, no or not able to examine/missing tooth. Depth of periodontal pockets was measured with a ball-ended WHO periodontal probe at four points (distal angle, midpoint of the buccal site, midpoint of the lingual site and mesial angle) and recorded by tooth according to the deepest probing pocket depth (PPD) as no pocket, pocket $\geq 4-5 \mathrm{~mm}$ or not able to examine. The examination was performed according to the asepsis of dental principles and ethical guidelines.

\section{Pharmacist interview}

A pharmacist interviewed the participants and recorded each prescription and non-prescription medication and complementary and alternative medicine supplements used regularly, as needed within a week or in the past on the basis of the interview, medication lists, packages and prescriptions at the baseline. All medications were encoded using the Anatomic Therapeutic Chemical (ATC) classification (WHO) [30]. Additionally, strengths, quantity, timing, indications and possible adverse reactions and problems were recorded. The medication list from the locally used electronic medical record (EMR) was gathered from the same day as the in-home interview.

\section{Intervention}

The NutOrMed study included nutritional and oral health interventions. On the basis of a blood test, MNA and nutritional history, the nutritionist developed a plan for individualised nutritional care and discussed it with the participant and her/his nurse or family members (Table 1). The objective of nutritional care was not to change the participants' food habits completely but to correct possible inadequacies in their diet. The main objective was to recommend food items that they were familiar with and which were already part of their daily diet. If the participants seemed unable to increase the energy and/or protein of their diet, daily complementary dietary drinks were recommended to them. In addition, the intervention persons were advised to take a $20-\mu \mathrm{g}$ vitamin $\mathrm{D}$ supplement daily. After the dental hygienist interview and oral health examination, the participants in need were targeted for oral health intervention. The intervention included individualised instructions on either dental hygiene, denture hygiene, cleaning of the oral mucosa or treatment for dry mouth.

The participants were re-examined by home care nurses, nutritionists and dental hygienists 6 months after the baseline interviews to evaluate the effectiveness of

Table 1 The inclusion criteria for nutritional interventions

\begin{tabular}{ll}
\hline & Inclusion criteria \\
\hline Intervention 1 & EM or risk of EM (MNA score $<24)$ \\
Intervention 2 & PM or risk PM plasma albumin $<35 \mathrm{~g} / \mathrm{L}$ \\
Intervention 3 & PEM or risk of PEM (MNA score \\
& $<24$ and plasma albumin $<35 \mathrm{~g} / \mathrm{L}$ \\
\hline
\end{tabular}

$E M$ energy malnutrition, $P M$ protein malnutrition, $P E M$ protein-energy malnutrition, MNA Mini Nutritional Assessment 
the interventions (Fig. 1). The participants in both the intervention and control groups had the same examinations with the exception of the nutritional and oral health intervention.

\section{Outcome measures}

The primary outcome measures are the following: (1) improvement in nutritional status measured by MNA test, laboratory tests, change in weight and BMI and nutrient intake based on a 24-h dietary recall; (2) improvement in oral health status measured by clinical oral health examination and in-home interview (self-reported oral health); and (3) agreement between in-home interview and EMR regarding medication. The measurements of outcomes 1-2 were done at the baseline and after the 6-month follow-up. Drug use was evaluated only at the baseline.

The secondary outcome measures are based on the results of the self-reported questionnaire: $\operatorname{mood}(\mathrm{GDS}-15)$, activities of daily living (Barthel Index) and instrumental activities (Lawton and Brody scale), blood pressure (mmHG) and chair stand test (s).

\section{Statistical analysis}

Analyses were performed using SPSS version 19.0 (SPSS, Inc., Chicago, IL, USA). The change that occurred during the intervention period was calculated for each outcome measure. Where the distribution of the change was approximately normal, an independent $t$ test was used to compare the groups. When the data were not normally distributed, a Mann-Witney $U$ test was used. Statistical comparisons between the groups were made using a chisquare test or $t$ test, with 0.05 considered significant. The results are expressed as means or frequencies with standard deviations (SD) or percentiles. A regression analyses was used to measure the effectiveness of the intervention over the follow-up period.

\section{Ethics approval}

The study protocol was approved by the Research Ethics Committee of the Northern Savo Hospital District, Kuopio, Finland.

\section{Discussion}

The present study describes the study protocol of the first intervention study examining the relationship between nutritional status, oral health and medication use in a home care population.

During the last few years, living at home has been preferred over residential care $[2,31]$. Providing home care services that meet the needs of community-dwelling older people is an increasingly pertinent issue. Already numerous countries, including Finland, have observed a substantial rise in expenditure and demand for home care services, which is predicted to continue. Our study provides evidence on the factors associated with nutrition, oral health and medication use that may compromise the ability to stay at home.

The strengths of this study are its multidisciplinary approach, the diversity of the measures, including interviews and clinical examinations, as well as the multiinterventions targeting those in need. The findings of this study are directly applicable to real life, allowing health care professionals to focus on nutrition, oral health care and medication use among older people. In addition, the present study had no exclusion criteria regarding age and morbidity or the cognitive status of home care clients.

\section{Competing interests}

The authors declare that they have no competing interests.

\section{Authors' contributions}

MT, KA-H and IN drafted the manuscript and participated in the analysis and interpretation of the data. RA, KK, LS and SH were involved in designing and revising the manuscript. All the authors read and approved the final manuscript.

\section{Acknowledgements}

This study was funded by the University of Eastern Finland, Faculty of Health Sciences. IN received funding from the North Savo Regional Fund.

\section{Author details}

${ }^{1}$ Kuopio Research Centre of Geriatric Care, University of Eastern Finland, P.O.B 1627, Fl-70211 Kuopio, Finland. ${ }^{2}$ School of Pharmacy, Faculty of Health Sciences, University of Eastern Finland, P.O.B 1627, FI-70211 Kuopio, Finland. ${ }^{3}$ Institute of Dentistry, School of Medicines, Faculty of Health Sciences, University of Eastern Finland, P.O.B 1627, FI-70211 Kuopio, Finland. ${ }^{4}$ Research Centre for Comparative Effectiveness and Patient Safety (RECEPS), University of Eastern Finland, P.O.B 1627, Fl-70211 Kuopio, Finland. ${ }^{5}$ Kuopio University Hospital, Department of Oral and Maxillofacial Surgery, Kuopio University Hospital, P.O. Box 100FI-70029 KYS, Kuopio, Finland.

Received: 12 August 2014 Accepted: 3 July 2015

Published online: 17 July 2015

\section{References}

1. Eurostat. Regional population projections EUROPOP2008: Most EU regions face older population profile in 2030. Statistics in focus 1/2010. 2010. http:// ec.europa.eu/eurostat/documents/3433488/5564440/KS-SF-10-001-EN.PDF/ d5b8bf54-6979-4834-998a-f7d1a61aa82d.

2. Genet N, Boerma WG, Kringos DS, Bouman A, Francke AL, Fagerstrom C, et al. Home care in Europe: a systematic literature review. BMC Health Serv Res. 2011;11:207-6963-11-207.

3. Turjamaa R, Hartikainen S, Pietila AM. Forgotten resources of older home care clients: focus group study in Finland. Nurs Health Sci. 2013;15:333-9.

4. National Institute for Health and Welfare. Institutional care and housing services in social care 2013. http://www.julkari.fi/bitstream/handle/10024/ 116944/Tr27 14.pdf?sequence=4.

5. Furuta M, Komiya-Nonaka M, Akifusa S, Shimazaki Y, Adachi M, Kinoshita T, et al. Interrelationship of oral health status, swallowing function, nutritional status, and cognitive ability with activities of daily living in Japanese elderly people receiving home care services due to physical disabilities. Community Dent Oral Epidemiol. 2013;41:173-81.

6. van der Putten JJ, Hobart JC, Freeman JA, Thompson AJ. Measuring change in disability after inpatient rehabilitation: comparison of the responsiveness of the Barthel index and the Functional Independence Measure. J Neurol Neurosurg Psychiatry. 1999;66:480-4.

7. Nykanen I, Lonnroos E, Kautiainen H, Sulkava R, Hartikainen S. Nutritional screening in a population-based cohort of community-dwelling older people. Eur J Public Health. 2013;23:405-9. 
8. Soini $H$, Routasalo $P$, Lauri S. Nutrition in patients receiving home care in Finland: tackling the multifactorial problem. J Gerontol Nurs. 2006;32:12-7.

9. Holmen A, Stromberg E, Hagman-Gustafsson ML, Wardh I, Gabre P. Oral status in home-dwelling elderly dependent on moderate or substantial supportive care for daily living: prevalence of edentulous subjects, caries and periodontal disease. Gerodontology. 2012;29:e503-511.

10. Syrjala AM, Pussinen PI, Komulainen K, Nykanen I, Knuuttila M, Ruoppi P, et al. Salivary flow rate and risk of malnutrition-a study among dentate, community-dwelling older people. Gerodontology. 2012;30:270-5.

11. Fialova D, Topinkova E, Gambassi G, Finne-Soveri H, Jonsson PV, Carpenter I, et al. Potentially inappropriate medication use among elderly home care patients in Europe. JAMA. 2005;293:1348-58.

12. Jyrkka J, Enlund H, Lavikainen P, Sulkava R, Hartikainen S. Association of polypharmacy with nutritional status, functional ability and cognitive capacity over a three-year period in an elderly population. Pharmacoepidemiol Drug Saf. 2011;20:514-22.

13. Komulainen $K$, Ylostalo $P$, Syrjala AM, Ruoppi $P$, Knuuttila M, Sulkava R, et al. Preference for dentist's home visits among older people. Community Dent Oral Epidemiol. 2012:40:89-95.

14. Onder G, Carpenter I, Finne-Soveri H, Gindin J, Frijters D, Henrard JC, et al. Assessment of nursing home residents in Europe: the Services and Health for Elderly in Long TERm care (SHELTER) study. BMC Health Serv Res. 2012;12:5-6963-12-5.

15. Nykänen I, Rissanen TH, Sulkava R, Hartikainen S. Effects of individual dietary counseling as part of a Comprehensive Geriatric Assessment (CGA) on nutritional status: a population-based intervention study. J Nutr Health Aging. 2014;18:54-8.

16. Crum RM, Anthony JC, Bassett SS, Folstein MF. Population-based norms for the Mini-Mental State Examination by age and educational level. JAMA. 1993:269:2386-91.

17. Sheik J, Yesavage J. Geriatric Depression Scale (GDS): recent evidence and development of a shorter version. Clin Gerontol. 1986;5:165-72.

18. Lawton MP, Brody EM. Assessment of older people: self-maintaining and instrumental activities of daily living. Gerontologist. 1969;9:179-86.

19. Groll DL, Heyland DK, Caeser M, Wright JG. Assessment of long-term physical function in acute respiratory distress syndrome (ARDS) patients: comparison of the Charlson Comorbidity Index and the Functional Comorbidity Index. Am J Phys Med Rehabil. 2006;85:574-81.

20. Tikkanen P, Nykanen I, Lonnroos E, Sipila S, Sulkava R, Hartikainen S. Physical activity at age of 20-64 years and mobility and muscle strength in old age: a community-based study. J Gerontol A Biol Sci Med Sci. 2012;67:905-10.

21. Covinsky KE, Covinsky MH, Palmer RM, Sehgal AR. Serum albumin concentration and clinical assessment of nutritional status in hospitalized older people: different sides of different coins? J Am Geriat Soc. 2002:50:631-7.

22. Kyle UG, Unger P, Mensi N, Genton L, Pichard C. Nutritional status in patients younger or older than $60 \mathrm{y}$ at hospital admission: a controlled population study in 995 subjects. Nutr. 2002;18:463-9.

23. Potter MA, Luxton G. Prealbumin measurement as a screening tool for protein calorie malnutrition in emergency hospital admissions: a pilot study. Clin Invest Med. 1999;22:44-53.

24. Guralnik JM, Simonsick EM, Ferrucci L, Glynn RJ, Berkman LF, Blazer DG, et al. A short physical performance battery assessing lower extremity function: association with self-reported disability and prediction of mortality and nursing home admission. J Gerontol. 1994;49:M85-94.

25. Guigoz Y, Vellas B, Garry PJ. Assessing the nutritional status of the elderly: the Mini Nutritional assessment as part of the geriatric evaluation. Nutr Rev. 1996;54:S59-65.

26. Vellas B1, Villars $H$, Abellan G, Soto ME, Rolland Y, Guigoz Y, et al. Overview of the MNA-its history and challenges. J Nutr Health Aging. 2006;10:456-63.

27. Kondrup J1, Allison SP, Elia M, Vellas B, Plauth M, Educational and Clinical Practice Committee, et al. ESPEN guidelines for nutrition screening 2002. Clin Nutr. 2003;22:415-21.

28. WHO. Oral Health Surveys. Basics Methods. 4th ed. Geneva: WHO; 1997.

29. Silness J, Löe H. Periodontal disease in pregnancy. II. Correlation between oral hygiene and periodontal condition. Acta Odontol Scand. 1964;22:121-35.

30. WHO. Anatomical Therapeutic Chemical (ATC) classification system: guidelines for ATC classification and DDD assignment. Oslo: WHO Collaborating Centre for Drug Statistics Methodology; 2013. http:// www.whocc.no/atc_ddd_methodology/purpose_of_the_atc_ddd_system/.
31. Finlex. Laki ikääntyneen väestön toimintakyvyn tukemisesta sekä iäkkäiden sosiaali- ja terveyspalveluista (Vanhuspalvelulaki). http://www.finlex.fi/fi/laki/ ajantasa/2012/20120980.

\section{Submit your next manuscript to BioMed Central and take full advantage of:}

- Convenient online submission

- Thorough peer review

- No space constraints or color figure charges

- Immediate publication on acceptance

- Inclusion in PubMed, CAS, Scopus and Google Scholar

- Research which is freely available for redistribution 